\title{
Influence of Clinical Variables to Cognitive Function in Diabetic Patients
}

\author{
Rajesh Venkataraman ${ }^{* 1}$, Kannadasan $\mathrm{T}^{1}$, Anand vijayakumar P. R ${ }^{2}$, \\ Balamurugan Ramanathan ${ }^{3}$ \\ ${ }^{1}$ Anna University, Chennai, \\ ${ }^{2}$ JSS College of Pharmacy, Ooty (JSS Univeristy, Mysore), \\ ${ }^{3}$ Kovai Diabetes Speciality Centre \& Hospital, INDIA.
}

\begin{abstract}
The objective of the research was to investigate the cognitive effects of clinical variables associated with health care in diabetes management in their course over time. The results of the studies show that diabetes and its complications along with socio demographic factors impart a significant impairment in cognitive domains. Thus far no long term large prospective study has specifically examined for the possible effect modification of cognition in diabetic patients in India. With Diabetes becoming an emerging threat and a burden to the country's economy it is vital that screening for cognitive dysfunction; be made as an integral part of the assessment process for subjects with diabetes mellitus. Evidence of significant decrease in cognitive functioning of diabetic patients with regard to Age, Gender and social habits paves way for need of a remarkable change in diabetes management. Future studies should aim to employ longitudinal designs to clarify more carefully the relationship between diabetes and cognitive function and better identify risk factors for developing cognitive dysfunction. The study carried out on 500 diabetic patients of various socio demographic characteristics over a period of eighteen months with Mini Mental State Examination (MMSE scale). The research carried to investigate the cognitive effects of clinical variables associated with health care in diabetes management in their course over time. The results of the studies show that diabetes and its complications along with clinical variables factors impart a significant impairment in cognitive domains.
\end{abstract}

\section{Introduction}

Diabetes mellitus is a group of common metabolic disorders. All types of diabetes mellitus are characterized by hyperglycemia, caused by defective insulin secretion, defective insulin action, or both. A simple and still formally correct way of subdividing most diabetic diseases is into type 1 and type 2 subcategories (Gavin et al., 2000).

The increasing incidence of diabetes mellitus in the industrialized world represents one of the most serious challenges facing the medical profession today. Diabetes and its complications have been estimated to cost $>\$ 130$ billion every year in the United States, and this figure is likely to grow as a sedentary lifestyle and aging population drive up the incidence and prevalence of disease(Hogan et al.,2002). The figures underscore the massive economic and societal burden associated with diabetes and its complications, and clearly indicate the need for action.

Of the 2 types of diabetes, type 1 is the least common, accounting for $-10 \%$ of total cases. It is characterized by insulin deficiency following selective destruction (usually mediated by the immune system) of insulin-producing beta cells in the pancreas, and its treatment involves administration of exogenous insulin. Type 2 diabetes is a heterogeneous group of disorders that involve impairment of the insulin-secretory response to glucose and insulin resistance (eg, decreased effectiveness of insulin in stimulating glucose uptake by skeletal muscle and limiting hepatic glucose production). Type 2 diabetes accounts for $90 \%$ of all diagnosed cases of diabetes and usually presents in adults. Several options are available for the treatment of type 2 diabetes, including diet, exercise, oral antidiabetic agents, and insulin. However, the hallmark of type 2 diabetes is successive movement through oral treatments, with many patients ultimately requiring insulin as the disease progresses.

\section{Experimental Methods}

Data collection forms were designed with respect to age, sex, education and income status. 500 patients of varying age groups [below 30, 30 to 60, above 60] were being recruited for the study. Study was conducted for the cognitive function through Mini Mental Test Score Examination. Study was conducted for the extent of adherence to treatment recommendations. Interpretation of the test results of patients under varying demographic data. Concluding the effects of adherence, drugs, age, sex, education and income status. 


\section{Inclusion criteria}

$>$ Patients willing to participate in the study.

$>$ Patients suffering from diabetes mellitus.

$>$ Normal subjects willing to participate in the study.

\section{Exclusion criteria}

$>$ Patients below 20 years and above 90 years.

$>$ Patients not willing to participate in the study.

$>$ Mentally challenged patients.

\section{Sources of data}

$>$ The various resources used for the collection of data include the following:

$>$ Interview with the patients.

$>$ Mini mental state examination form (shown in AnexureIV and AnexureV).

$>$ Inpatients data collection form (shown in Anexure III).

$>$ Patient case history.

$>$ Patient treatment schedule charts.

\section{Ethical clearance}

The study was approved by the Ethics Committee of M.S. Chellamuthu Trust and Research Foundations, Madurai, Tamil Nadu, India. Patients were informed that the information they provided was confidential and would be presented only as group information without any identifying characteristics. Written informed consent was provided by all patient participants prior to entry into the study (shown in Anexure II).The approval from the conserned ethics committee has been provided in the Anexure I.

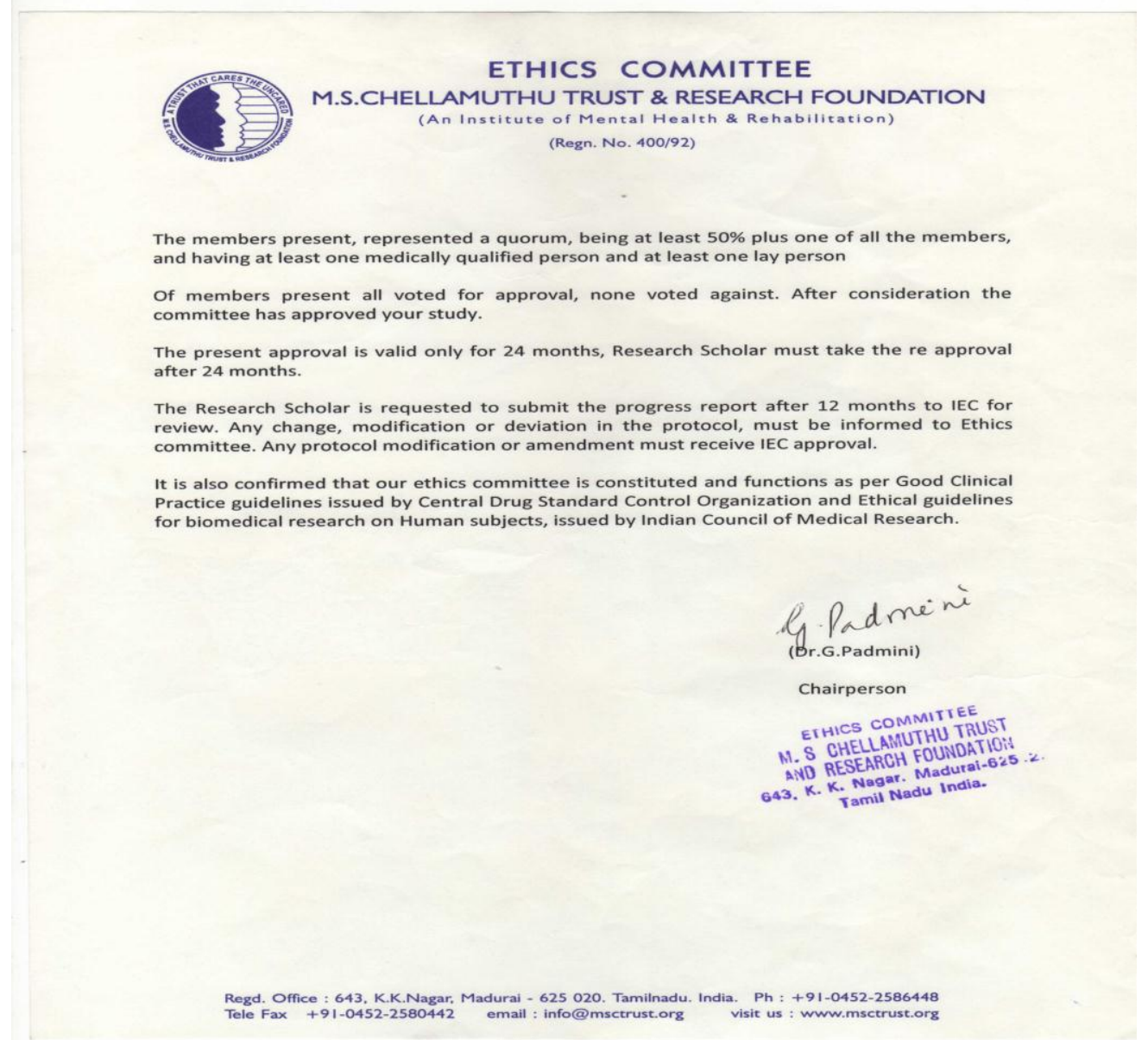

\section{Data and safety monitoring}

This research involves no more than minimal risk to participants. There will be no participant names on the study forms and all forms will be stored in a locked file cabinet in the investigator's office. 


\section{Human subjects instructions}

The population for this study includes 500 participants of varying demographic population. The population is comprised of men and women in and around Coimbatore. It is assured that anyone not willing to participate in the study would not be enrolled in the study.

\section{Recruitment plan}

All patients fulfilling the inclusion criteria and who are willing to participate in the study were being invited to participate in the study. It was estimated that completion of the survey, MMSE exam, and demographic form will take approximately 15 minutes.

\section{Potential risks}

This research presents no more than minimal risk to its participants.

\section{Risk reduction}

All surveys will be completed anonymously and are returned in unmarked envelopes. Because the nature of the survey questions is not sensitive and no identifying information is collected, this poses almost no risk to participants. All data will be maintained by the Scholar. All data is completely anonymous, as there is no link between names and responses. Surveys will be stored by the scholar. Computer data files will be stored on a secure computer.

\section{Confidentiality}

All data will be maintained by the scholar and is accessible to the concern guides only. Survey data will not include personal data of the patients.

\section{Risk/benefit}

The minimal risk to participants is reasonable in relation to the benefit of increased knowledge. The results of this study are important in understanding cognition with regard to diabetes in help framing a better diabetes management.

\section{Research Procedure}

- Patients satisfying the study criteria were enrolled after their informed consent.

- Cognitive impairment examination was conducted in the subjects.

- A patient was considered a drop out provided the patient completely fails to attend any of the examinations.

\section{Measures of cognitive performance}

Cognitive functioning in the cohort was measured using the MMSE scale (MINI MENTAL STATE EXAMINATION).The scale was administered on every subject and data recorded from baseline to the second follow up. The data were statistically analysed for the influence of gender, age and social habits on cognitive functioning in diabetic cohort (shown in Anexure VI). A pilot study was done prior to the study, pilot study was performed to standardise the scale as per the study environment.

\section{Statistical analysis}

A statistical power analysis was performed before the study start. It was calculated that 500 patients would be enough to detect 1 significant differences in cognitive functioning ( $>5$ points/dimension) in the OPTIMAL-study, with a power of $80 \%$. In order to compensate for a potential loss of $10 \%$ of patients during the course of the study, we intended to include 600 patients at the start of the study.

Statistical analysis was done using SPSS V10 on windows xp plat form. Means of continuous measures across categorical variables were tested using t-Test and analysis of variance (ANOVA).

\section{IMPACT OF CLINICAL VARIABLES ON COGNITIVE FUNCTIONING}

As per the correlation analysis on clinical variables frequency sweating exercise (FRSWES),consumption of food at regular intervals (CONFRI), following recommended diet (Follow diet) are positively significantly and correlated with base, $1^{\text {st }}$ and II $^{\text {nd }}$ follow up. Thereby proving that these variables directly impart there effect on cognitive functioning. 


\section{Patient enrollment}

\section{Result And Discussion}

Among the 600 patients screened 16 patients did not full fill the inclusion criteria. A baseline was marked with 574 patients; the data were collected from patients as per ethical consideration after receiving the concern form. After six months interval the 554 patients were studied with the same questionnaire as used in the baseline with similar environmental factors as that of the baseline. The remaining 20 patients failed to appear for the follow up. The study was repeated after the prescribed duration of six months interval with the same cohort and data were recorded, 500 patients completed the study. The diabetic population consisted of 100 patients who had completed primary education, 138 secondary, 104 higher secondary, 140 patients who had attained a degree or diploma and 18 illiterate patients.

\section{Pilot study}

A pilot study was done prior to patient enrollment to stabilize the MMSE scale thereby enhancing the reliability and validity as per the research environment.

\section{A and B Reliability and validity}

The responses were scored and these two sets of scores (control and test group) of each test were treated through product moment correlation for working out the reliability co-efficient. Test of significance was computed to the reliabilities and validities of the tests and scales by employing the formula $t=r \times \sqrt{ }(n-2) / 1-r^{2}$ as suggested by Edwards. The reliability and validity are statistically significant. They are furnished in the following table: Using test-retest method the reliability and validity of the tool was worked out.

Table 1: A Reliability co-efficient and test of significance for the study

\begin{tabular}{cccccc}
\hline Sl.No & Test & N & Reliability Co-efficient & Test of Significance & Level of Significance \\
\hline 1. & TMMSE Score & 100 & 2.42 & 2.914 & 0.001 \\
\hline
\end{tabular}

Table B Validity co-efficient and test of significance for the study

\begin{tabular}{ccccccc}
\hline Sl.No & Test & $\mathbf{N}$ & $\begin{array}{c}\text { Validity } \\
\text { efficient }\end{array}$ & $\begin{array}{c}\text { Co- } \\
\text { Significance }\end{array}$ & $\begin{array}{c}\text { Level of } \\
\text { Significance }\end{array}$ \\
\hline 1. & TMMSE Score & 100 & 2.84 & 2.596 & 0.001 & \\
\hline
\end{tabular}

Table 3: Correlation between MMSE scores and clinical variables

\begin{tabular}{cccc}
\hline Clinical Variables & TMMSE Base & TMMSE Ist Follow up & TMMSE II $^{\text {nd }}$ Follow up \\
\hline SBP & -0.015 & -0.012 & -0.004 \\
DBP & -0.057 & -0.047 & -0.048 \\
FBS & 0.013 & 0.009 & 0.006 \\
PPBS & 0.037 & 0.051 & 0.059 \\
STCHOL & -0.051 & -0.060 & -0.060 \\
LDL & -0.052 & -0.051 & -0.043 \\
HDL & -0.080 & -0.080 & -0.068 \\
CRE & 0.020 & 0.020 & 0.039 \\
HbA $A_{1 c}$ & -0.039 & -0.038 & -0.031 \\
FRSWEX & $0.192^{* *}$ & $0.189^{* *}$ & $0.191^{* *}$ \\
CONFRI & $-0.158^{*}$ & $-0.168^{* *}$ & $-0.169^{* *}$ \\
KNHOW & -0.099 & -0.100 \\
Follow diet & -0.088 & $-0.179 * *$ & $-0.191^{* *}$ \\
CVSEVT & $-0.184^{* *}$ & 0.034 & 0.030 \\
SENTES & 0.025 & 0.020 & 0.019 \\
MED & 0.019 & 0.022 & - \\
BMI & 0.024 & 0.014 & - \\
\hline & 0.034 & Correlation is significant at the 0.05 level & \\
& $* *$ Correlation is significant at the 0.01 level &
\end{tabular}




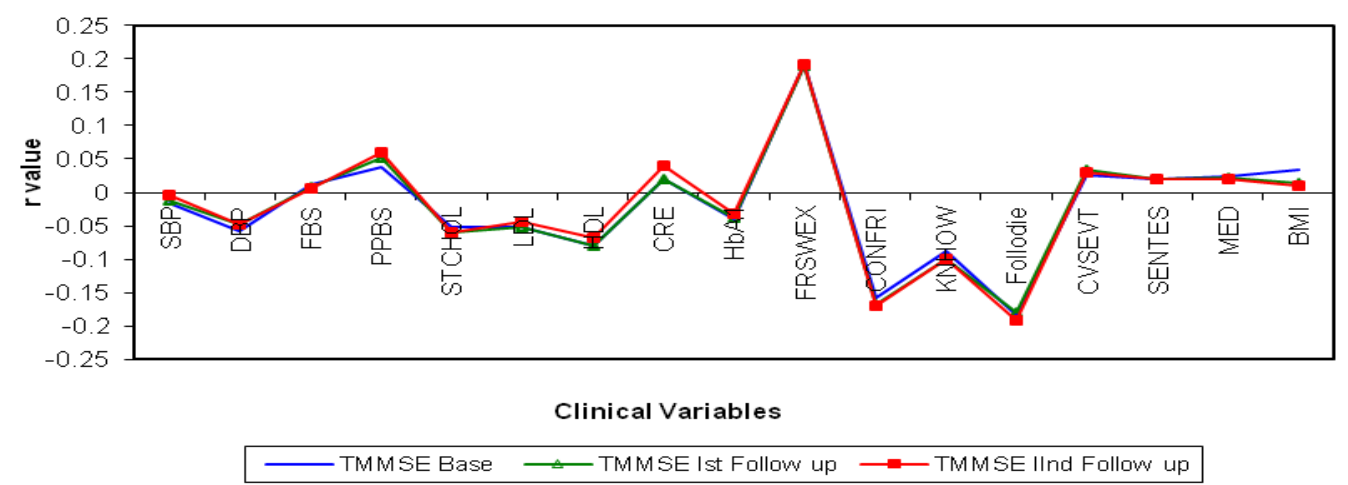

Figure 1: correlations between MMSE scores and clinical variables

\section{Conclusion}

Evidence of significant decrease in cognitive functioning of diabetic patients with regard to clinical variables impart that have yet to be identified crucial in management of diabetes and its complications. The rising prevalence of diabetes poses a major clinical, economical and social burden to developing country like India. With long term complications of the diabetes being extremely alarming the health care requires a renewal with regard to individualized patient care in diabetes management with regard to cognitive dysfunction a potential threat among diabetic individuals.

\section{References}

[1]. Aevarsson.O, Skoog I (2000). "A longitudinal population study of the minimental state examination in the very old: relation to dementia and education", Dement Geriatr Cogn Disord, vol. 11, pp.166-75.

[2]. Albert MS, Moss MB (1988). "Geriatric Nemopsychology", New York: Guilford Press, pp.33-56.

[3]. Alexopoulos.P, Grimmer.T, Perneczky.R, Domes.G and Kurz.A (2006). "Progression to dementia in clinical subtypes of mild cognitive impairment”. Dement Geriatr Cogn Disord, vol. 22 , no.1, pp.27-34.

[4]. Andreasen.N, Vanmechelen.E, Vanderstichele.H, Davidsson.P and Blennow.K (2003). "Cerebrospinal fluid levels of total-tau, phospho-tau and A beta 42 predicts development of Alzheimer's disease in patients with mild cognitive impairment”. Acta Neurol Scand issue, 179 , pp.47-51.

[5]. Anstey, K., Christensen, H. (2000), "Education, activity, health, blood pressure and apolipoprotein E as predictors of cognitive change in old age: a review”. Gerontology, vol.46, pp. 163-177.

[6]. Anthony J.C, LeResche L, Niaz .U (1982). "Limits of the Mini-Mental State as a screening test for dementia and delirium among hospital patients". Psychol Med, vol.12, pp.397-408.

[7]. Apter, N.S., Halstead, W.C, Heimburger.R.F. (1951). "Impaired cerebral functions in essential hypertension". American Journal of Psychiatry, vol .107, pp.808-813.

[8]. Arvanitakis Z, Wilson RS, Li Y, Aggarwal NT, Bennett DA (2006). "Diabetes and function indifferent cognitive systems in older individuals without dementia" Diabetes Care.vol.29, pp.560-565.

[9]. Arvanitakis, Z., Wilson, R. S., Bienias, J. L., Evans, D. A., and Bennett, D. A (2004). "Diabetes Mellitus and Risk of Alzheimer Disease and Decline in CognitiveFunction". Archives of Neurology, vol.61.no.5,pp. 661-666.

[10]. Assisib. M., Alimentib.F., Maceliab. S., Di Pietroab. G., Lalloniab. P., Montera., (1996). "Diabetes and cognitive function: preliminary studies". Archives of Gerontology and Geriatrics,vol .22, no. 1, pp. 229-232.

[11]. Backman L, MacDonald S.W.S (2006). "Death and cognition desynthesis and outlook". Eur Psychol, vol.11.no, 44, pp.224-35.

[12]. Baker L. and BARCAI A (1970). "Psychosomatic aspects of diabetes mellitus". In Modern Trends in Pspchosomntic Medicine (Edited by 0. W. HILL). Butterworths, London.

[13]. Barclay, L. L., Weiss, E. M., Mattis, S., Bond, O., and Blass, J. P (1988). "Unrecognizedcognitive impairment in cardiac rehabilitation patients". Journal of the AmericanGeriatrics Society, vol.36, issue.1, pp. 22-28.

[14]. Beasers. B (1956). "Teaching the diabetic patient". Dioberes ,vol.5, pp. 146-149.

[15]. Becker M.H (1976). "Sociobehavioural determinants of compliance". In Compliance with Therapeutic Regimens (Edited by D. L. Sacket and R. B. Haynes) Johns Hopkins Press. London,

[16]. Biessels, G. J (1999). "Cerebral complications of diabetes: clinical findings and pathogenetic mechanisms".The Netherlands Journal of Medicine, vol.54, no.2, pp. 35-45.

[17]. Blennow.K, Wallin.A, Uhlemann.C, Gottfries.C (1991). "White-matter lesions on CT in Alzheimer patients: relation to clinical symptomatology and vascular factors”. Acta Neurol Scand, vol.83, pp.187-193. 